\title{
Location Provider with Privacy Using Localized Server and GPS
}

\author{
Pintu.R \\ Department of CSE \\ Bharath University \\ Chennai, India
}

\author{
Rahul Gupta \\ Department of CSE \\ Bharath University \\ Chennai, India
}

\author{
G.Michael \\ Department of CSE \\ Bharath University \\ Chennai, India
}

\begin{abstract}
:
Maps are an essential part of any handheld device and use constantly used for navigation and other resources by application for providing location based data which can be used for customized examination outcomes, however these data are conservatively stowed on a L.B.S Server which are susceptible to attacks and misuse as these data's are not usually have any significant security so these data's can be sold or misused by some other parties. We try to eliminate the problem as well as provided added functionality to the conventional maps by providing a customizable map which has the added functionality of offline use mode in addition to the online mode.
\end{abstract}

Keywords: GPS, MySQL Server, Maps, Android, SQLite

\section{INTRODUCTION}

Information agents are software products for assisting and guiding users to reach the goal of information retrieval. Up to now, however, most of Web information agent systems are closely related to the traditional information equipment's that cannot directly apply to the modern mobile equipment resulting from the core part of information agent in ubiquitous environments [1], [2]. This study exactly focuses on how to design a ubiquitous interface agent with mobile equipment's in ubiquitous environments.[3], [4] Ubiquitous computing is a post-desktop model of human computer interaction in which information processing has been thoroughly integrated into everyday objects and activities. Cloud computing is a technique of Internet- ("cloud-") based development and use of computer technology. Furthermore, how to construct an interaction diagram of cloud computing for extensively and seamlessly entering related web information agent systems through modern mobile equipment's in ubiquitous environments is under our investigation.

\section{RELATED WORKS}

In this paper,A new ubiquitous informational agent system with the GPS and Bluetooth techniques in the Google Android platform and related interaction diagrams with Onto IAS in cloud computing environments was proposed in this paper. It also explains how GPS

Devices access location based services by connecting to the google cloud server. With the propagation of portable expedients, impulsive connections between co-located devices that do not know each other a priori will become commonplace.

In this paper[5],[6] we postulate that mobile devices that are positioned in close proximity may be able to derive a shared secret to secure their communication by monitoring fluctuations in the signal strength of existing ambient radio sources (GSM cell towers or Wi-Fi access points) in their general environment. We explore the probability of deriving location-based secrets and describe two approaches for how such a secret could be used to secure spontaneous communication. Derive location-based secrets is a hard problem because while the radio environment perceived by various devices in close proximity is comparable, it is not undistinguishable. Global Positioning System (GPS) technology is changing the way we work and play.

In this paper you can use GPS technology when you are driving, flying fishing, marine, climbing, one after the other, biking, running, or exploring. Here are just a few examples of how you can use GPS technology Know precisely how far you have run and at what pace while tracking your path so you can find your way home Get the closest location of your favorite restaurant when you are out-of-town Find the nearest airport or identify the type of airspace in which you are flying., In existing system though we have many web sites gateway, and the user can get data about the searched location in web sites The user can be able to access the location only in online these systems are not available to not all time. And also, this information does not reach people at the time of emergencies

In this paper, in existing system if the user search some location the high level data information will be displayed on the screen. Whenever user want to search the places user need to specify the location of that particular area. in existing system user can be able to access the information which is stored in the server. The problem is to implement security to the location based services for the GPS devices on android platform. This is done by using a server implemented along with the main server to authenticate users. By implementing server additional features like real time location services are also made available to the user.

\section{PROPOSED WORK}

In the Proposed System of completion, user makes the query to the main record through our application. In proposed system Ontology is also implemented in this Project for the Relative Key word Search. If user search some location through our application it's take the current location of the application user using GPS. As the user makes the query along with his GPS values to find out the Exact Location from the data base, this process the query and authenticate the user and then passes the query to the main database The main system maps the query with its database along with the Location, auxiliary this location based in turn is passed back to the user. In existing system only contain the high level data but in our project 
contain the deep level data because those data are collect from locally.

\section{EXPERIMENTAL SETUP AND DESIGN}

Using the GPS we locate the exact location of the user and the data is sent to the user. This data accessed by the user can be downloaded by the user and saved onto his device which can be used in offline mode after which search can be made in offline mode and LBS is not contacted thus location privacy is maintained. Using Wi-Fi and Dead Reckoning the locations in indoor location is predicted, where Wi-Fi based location prediction is used for indoor and dead reckoning is used to predict the location of user based on previously determined location. Now this downloaded data can be edited by user and user can add information

And shared via the app and people using the application can view the information instead of LBS open availability..

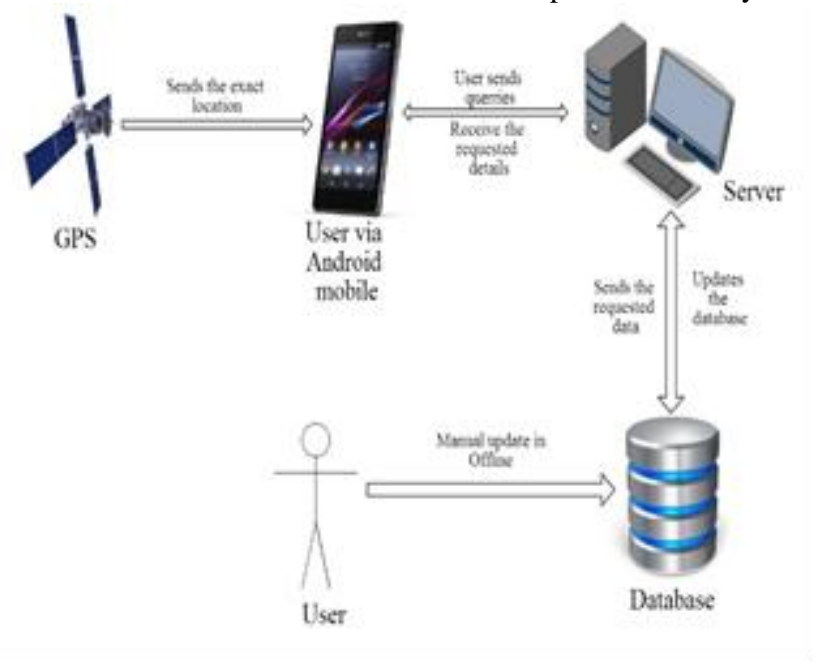

Figure. 1 System Architecture

\section{MODULE DESCRIPTION}

\subsubsection{Server Modules}

This module is mainly used to search the location of particular place in online from our server. The user can able to download the location to the local database consuming the server. After that user can be able to Examine the data in offline. In this server only the user can be able to upload the location to the server

\subsubsection{Searching in Offline and Online using Ontology}

In this module user can be able to search the location in online and in offline mode The current location of the user can be obtained using Indoor tracking and dead reckoning techniques Indoor tracking is a network of device used to wirelessly(WIFI,GPS) locate the user current location who is located inside the house . Dead reckoning is a procedure that is designed to guess current location based on the previously determined location 5.4.3 Add and Share location

In this module the user can be able to add the location details of particular place to the server by using data offloading method Data offloading method is used to deliver the original data's to the targeted server from the mobile device After adding the location to the server and server share the location to the other user who are all using this application

\subsubsection{Updating offline database}

In this modules the user can be able to update the location details from the server to the offline database In our application we are using SQLite as an offline database the user get the data from the centralized server and data will be send to the SQLite.

\section{IMPLEMENTATION AND WORKING}

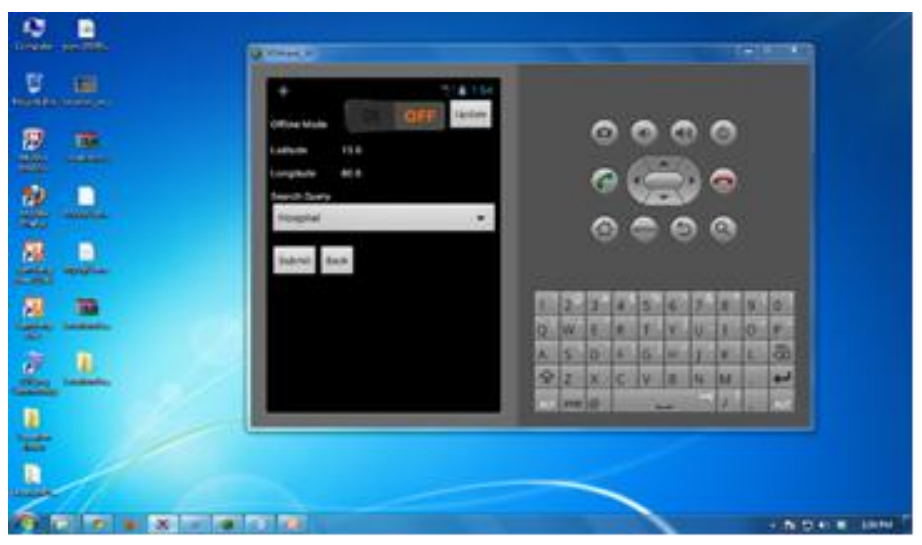

This is a screenshot of the android emulator running the map application on the system and shows the first module where we can make search on the map

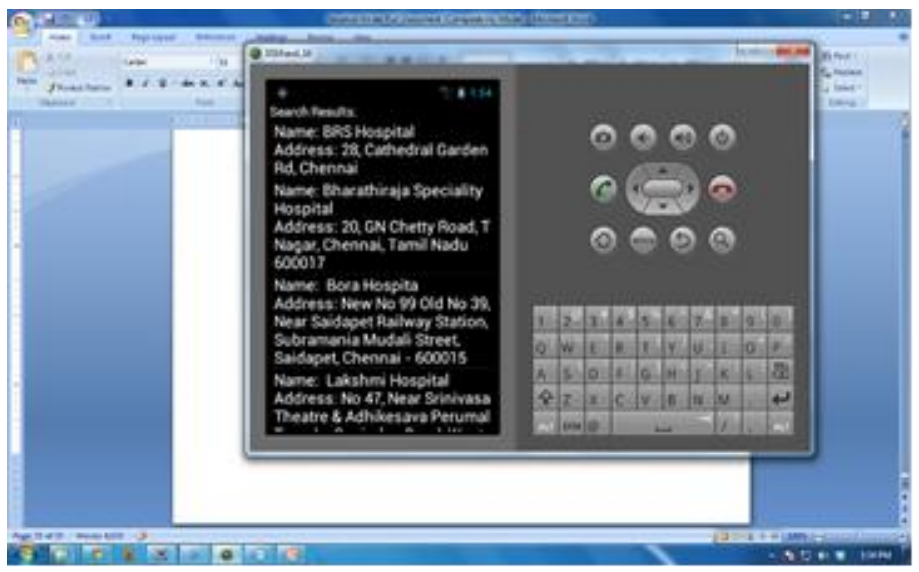

We see here in the second screenshot the search results for a search for hospitals made 


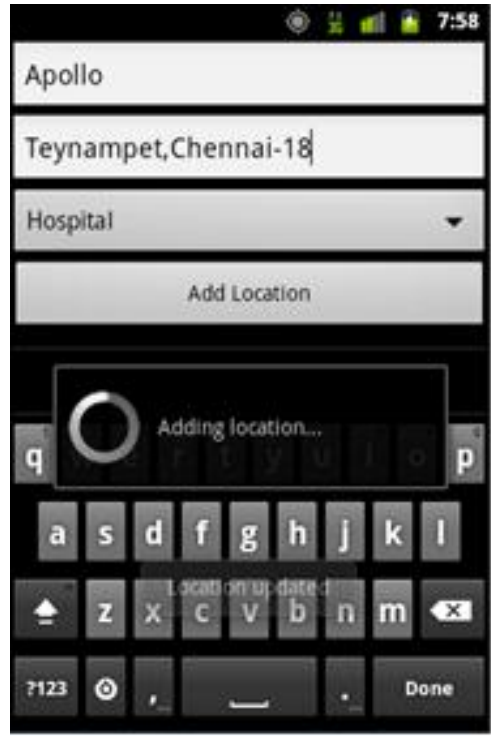

In this next screenshot we see the add location module we can edit the downloaded maps and customize and add locations to be shared

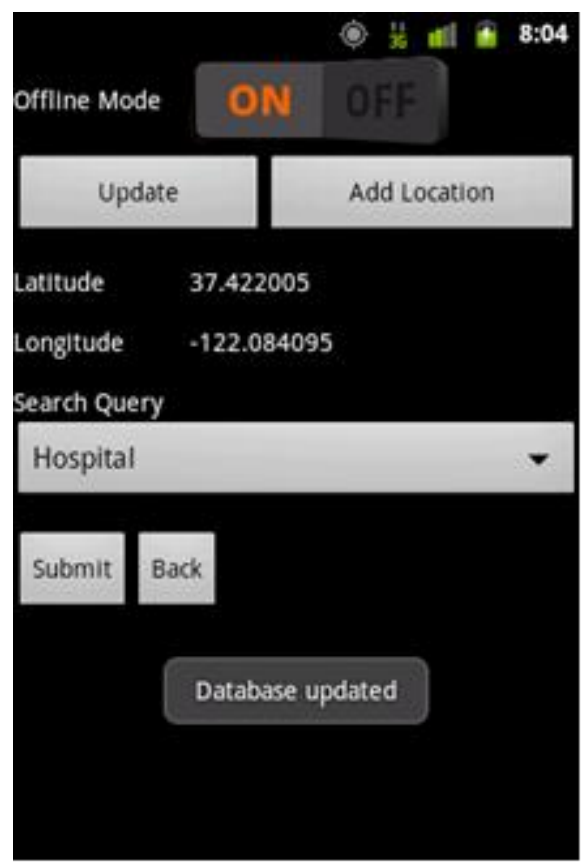

In this next screenshot we see how the offline mode is enabled to access the search in offline mode i.e. when not connected to internet still the locations can be accessed.

\section{ACKNOWLEDGMENTS}

We would like to extend our thanks to our Guide Prof Michael for his invaluable suggestions and guidance for the duration of the project and also to the all the people who have contributed for the project in any way.

\section{CONCLUSION}

We propose here a location provider which aims to provide the Location of user through a customizable map service for android devices which can be used in online as well as offline mode.

The request avoids the outdated LBS server based approach which is susceptible to attacks and misuse of data also using a customizable map a user can add functionality to maps and let other people see the updates made to the maps.

\section{REFERENCES}

[1] Alex Varshavsky, Antony Lamarca, Eyal Lara (2007)" Enabling secure and spontaneous communication between mobile devices using common radio environment". IEEE Workshop on Mobile Computing Systems and Applications (Hot Mobile), Tucson, AZ, February 2007.

[2] Garmin International, Inc., KS, USA (2000)"GPS guide for beginner"..

[3] Patridge.K and P.Golle (2008)"On using existing time-use study data for ubiquitous computing applications".UbiComp '08 Proceedings of the 10th international conference on Ubiquitous computing

[4] Yang.S.Y (2009) "A Study on Developing an OntologySupported Information Agent Shell". National Science council project, NSC-98-2221-E-129-012(NT\$:654,000), Taipei, Taiwan, 2009.

[5] Yang, Sheng-Yuan Lee, Dong-Liang(2011)"A new ubiquitous information agent system for cloud computing - example on gps techniques in google android platform". 01/2011; DOI:10.1109/COMPSYM.2010.5685438 In proceeding of: Computer Symposium (ICS), 2010 International.

[6] Yang.S.Y, K.W.Wu, C.M.Ni, Y.T.Lin and P.S.Kao (2010)" An ontology-supported ubiquitous interface agent for cloud computing - example on Bluetooth wireless technique with java programming". Proc. Of 2010 Conference on Information Technology and Applications in Outlying Islands, Kaohsiung, Taiwan, 2010 